\title{
Lidil
}

Revue de linguistique et de didactique des langues

\section{Lire et écrire les phénomènes de resyllabation : le rôle de la familiarité des séquences et de la fréquence des mots chez les enfants au $\mathrm{CP}$}

Writing and Reading in Presence of Resyllabified Phenomena: The Role of Item Frequency and Familiarity of Sequences in French First Graders

\section{Samantha Ruvoletto}

\section{OpenEdition}

\section{Journals}

Édition électronique

URL : http://journals.openedition.org/lidil/4162

DOI : $10.4000 /$ lidil.4162

ISSN : 1960-6052

Éditeur

UGA Éditions/Université Grenoble Alpes

Édition imprimée

ISBN : 978-2-37747-002-0

ISSN : $1146-6480$

\section{Référence électronique}

Samantha Ruvoletto, «Lire et écrire les phénomènes de resyllabation : le rôle de la familiarité des séquences et de la fréquence des mots chez les enfants au CP », Lidil [En ligne], 55 | 2017, mis en ligne le 02 mai 2017, consulté le 10 décembre 2020. URL : http://journals.openedition.org/lidil/4162 ; DOI : https://doi.org/10.4000/lidil.4162

Ce document a été généré automatiquement le 10 décembre 2020.

(C) Lidil 


\section{Lire et écrire les phénomènes de resyllabation : le rôle de la familiarité des séquences et de la fréquence des mots chez les enfants au CP}

Writing and Reading in Presence of Resyllabified Phenomena: The Role of Item Frequency and Familiarity of Sequences in French First Graders

\section{Samantha Ruvoletto}

\section{Introduction}

1 La langue française est perçue à l'oral comme un continuum dont les locuteurs peuvent avoir des difficultés à repérer les unités lexicales qui le composent, ou à déterminer leurs frontières. Comme l'a dit Delattre (1951), « le français enchaîne les mots, quitte à perdre de vue leurs limites. D'où l'impression unie, liée, que donne la chaîne parlée » (p. 62). Cette impression d'unité est causée par les caractéristiques linguistiques du français. L'accent de mot qui en détermine la prosodie fait que les mots sont plus difficilement discernables à l'oral (Encrevé, 1988; Blanche-Benveniste \& Jeanjean, 1987). De plus, l'organisation du français en structures syllabiques ouvertes (Delattre, 1965; Pulgram, 1970 ; Kaye \& Lowenstamm, 1984 ; Béchade, 1992 ; Di Cristo, 1998) rend les frontières des mots perméables aux influences contextuelles. Ainsi, nous pouvons citer des phénomènes phonologiques de resyllabation très communs à l'oral: la liaison, l'élision et l'enchainement.

2 Dans le cas de liaison exemplifié en (1), une consonne, dite consonne de liaison (CL), est produite et resyllabifiée au début de Mot2 s'il commence par une voyelle (ex. les[z]amis [le.za.mi] ; les garçons [le.gaь.s̃̃]). 
(1) Mot1 : les [le] + Mot2 : ami [ami] $\rightarrow$ les[z]ami [le.za.mi]

Dans le processus d'élision obligatoire, la voyelle finale du mot de gauche (Mot1) s'amuït devant un deuxième mot (Mot2) débutant par une voyelle. Ensuite la consonne qui était l'attaque du même mot devient l'attaque de Mot2, en conséquence de la chute d'un noyau vocalique, comme exemplifié en (2) :

(2) Mot1 : le [lə] + Mot2 : ours [uss] $\rightarrow$ l'ours [luss] où [ə] $\rightarrow \varnothing / \_V$

4 Pour l'enchainement, la consonne finale de Mot1, que nous pouvons appeler consonne fixe (CF) en utilisant la mention d'Encrevé (1983), se lie par enchainement à la voyelle initiale de Mot2 au moyen d'une resyllabation. Ainsi, la consonne, qui est en position de coda dans la dernière syllabe de Mot1, se retrouve dans la position d'attaque de la syllabe suivante comme il est possible de le voir en (3), où le [n] de une [yn] est la CF :

(3) Mot1 : une [yn] + Mot2 : orange [э.เãz] $\rightarrow$ un[n] orange [y.nว.ธã3]

Ces trois phénomènes sont toujours réalisés dans des séquences de mots déterminant +nom commun (Mot1+Mot2) lorsque le mot à droite (Mot2, le nom commun) commence par une voyelle. Cette étude se focalise sur ce contexte constitué par un déterminant, l'article, suivi d'un nom commun.

6 Si nous analysons les processus phonologiques à la base de ces phénomènes, nous pouvons remarquer que pour tous les trois, la consonne qui appartient au Mot1 est resyllabifiée au début de Mot2 (le [z] dans [le.zami], le [1] dans [luss] et le [n] dans [y.n ว.เãz]). Cette resyllabation implique un décalage entre les frontières syllabiques et les frontières lexicales de Mot2 et un masquage des frontières lexicales par les frontières syllabiques qui causent des difficultés dans la segmentation de la chaine orale chez les enfants francophones. Au début de l'acquisition de la langue orale, ces enfants utilisent les unités rythmiques qui caractérisent leur langue maternelle pour segmenter: les syllabes (Nazzi, Iakimova, Bertoncini, Fredonie \& Alcantara, 2006 ; Nazzi, 2008 ; Babineau \& Shi, 2011). En utilisant la syllabe comme unité fondamentale pour la segmentation, ils peuvent faire correspondre le début de la syllabe au début du mot et produire des erreurs qui reflètent une difficulté à déterminer la frontière gauche de Mot2. Plusieurs études chez les enfants francophones entre 2 et 5 ans (Wauquier \& Braud, 2005 ; Dugua, 2006; Nardy \& Dugua, 2011; Wauquier \& Shoemaker, 2013 ; Chevrot, Dugua, Harnois-Delpiano, Siccardi \& Spinelli, 2013) ont observé des productions caractérisées par l'intégration de la $\mathrm{CL}$ au début du nom (ex. de(s)[n]éléphants [de.ne.le.fõ] pour des[z]éléphants [de.ze.le.fơ]). Dans une moindre mesure, les chercheurs enregistrent également la complète omission

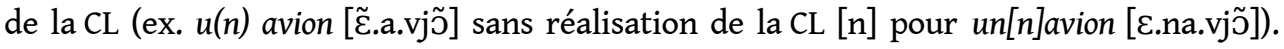
Dans ce type d'erreurs, la frontière gauche est identifiée et le Mot2 est isolé du mot qui précède. Ces erreurs de segmentation diminuent entre 5-6 ans (Dugua, Spinelli, Chevrot \& Fayol, 2009; Dugua \& Chabanal, 2006). Par ailleurs les seules erreurs qui persistent après 5 ans sont les omissions, c'est-à-dire lorsque la liaison n'est pas réalisée (Chevrot, Dugua, Harnois-Delpiano, Siccardi \& Spinelli, 2013).

7 Une difficulté à déterminer la frontière gauche de Mot2 a aussi été observée pendant l'apprentissage de la langue écrite chez des enfants au CE1 par Soum (1997) et par SoumFavaro, Gunnarsson \& Largy (2014). Dans deux analyses des productions écrites caractérisées par la liaison et l'enchainement, les chercheurs remarquent à plusieurs reprises la présence d'une erreur : la consonne resyllabifiée à l'oral au début de Mot2 est orthographiée comme son initiale (ex. <un gros sorage> pour un gros[z]orage ou <un nange> pour un[n]ange). Ces erreurs à l'écrit peuvent être expliquées par l'utilisation de 
l'unité syllabique en lecture et en écriture chez les enfants qui fréquentent l'école primaire (Goswami \& Bryant, 1990 ; Goswami, 1993, 1999; Colé, Magnan, \& Grainger, 1999 ; Kandel \& Valdois, 2006 ; Chetail, 2012). Pour apprendre à lire et à écrire, en effet, les enfants francophones ne peuvent pas utiliser seulement les correspondances graphème-phonème car dans leur langue, il est plus difficile d'accéder aux composantes mineures qui constituent les mots - les phonèmes - à partir de sa surface orale (Zigler \& Goswami, 2005). Pour cette raison, ils doivent faire référence à des unités plus grandes, comme la syllabe. En utilisant l'unité syllabique pour écrire, ils considèrent que la frontière orthographique gauche de Mot2 correspond à la frontière syllabique qu'ils entendent à l'oral. Ils orthographient donc le Mot2 comme un mot à consonne initiale (<nambulance>, <lavion> ou <zours>). L'utilisation de la syllabe en lecture ne semble pas compliquer la réalisation de la resyllabation. Comme montré par Dugua et Baclesse (2014) dans une tâche de lecture à voix haute au CE1, 96,5\% des liaisons obligatoires sont bien réalisées en lecture.

Jusqu'à présent, les études qui traitent des phénomènes de resyllabation à l'écrit prennent en considération les compétences des enfants à partir du CE1. De plus, elles ne tiennent pas compte des répercussions des données récoltées sur les méthodes pédagogiques. Cette étude cherche à décrire les processus d'écriture et de lecture des phénomènes de resyllabation dans une étape d'apprentissage de la littéracie encore inexplorée, le CP. Elle cherche également à mesurer l'influence de certaines variables (comme la fréquence) et à définir les stratégies pédagogiques qui doivent être mises en place pour réduire les erreurs et les difficultés dans la lecture et dans l'écriture des séquences déterminant+nom en liaison, élision et enchainement.

\section{Hypothèses}

9 Nous cherchons à voir si les enfants au $\mathrm{CP}$ ont des difficultés à identifier à l'écrit la frontière gauche de Mot2 ou à lire les séquences de Mot1+Mot2 en présence de phénomènes de resyllabation. Sur la base des données présentes dans la littérature, nous nous attendons à enregistrer des difficultés dans l'identification de la frontière gauche de Mot2 à l'écrit mais à ne pas observer d'erreurs dans l'enchainement des suites Mot1+Mot2 en lecture.

10 De plus, dans cette étude, nous cherchons à évaluer le rôle de la fréquence du Mot2 dans le lexique écrit des enfants et l'importance de la familiarité des séquences de Mot1+Mot2 présentées pendant l'apprentissage de la littéracie. Nous vérifierions si entrainer les enfants à lire et écrire une séquence d'un Mot1 suivi d'un Mot2 (ex. <les ours>) aide à orthographier leurs frontières et favorise le nombre de lectures correctes, ou s'il est plus efficace d'apprendre la forme écrite de Mot2 isolé (ex. <ours>) pour bien fixer la frontière gauche des mots à initiale vocalique à l'écrit. L'évaluation de la fréquence et de la familiarité nous permettra d'établir quelle méthode pédagogique est la plus efficace pour apprendre à lire et à écrire des séquences de mots en présence de liaison, d'élision et d'enchainement. 


\section{Méthode}

\subsection{Participants}

11 Nous avons contacté une école privée de Dunkerque, dans l'actuelle région des Hauts-deFrance, où la directrice a accepté que nous testions 43 enfants francophones (25 filles, 18 garçons ; âge moyen $M=6 ; 3$, écart-type $=0,5$ ) dans deux classes de CP pendant l'année scolaire 2013-2014. Les cours dans les deux classes sont assurés par deux professeures des écoles différentes. Cependant, les objectifs pédagogiques (les programmes, les méthodes, les tâches et le suivi des acquis attendus chez les enfants) sont communs, grâce à la communication quotidienne entre les deux professeures. De plus, toutes deux adoptent une méthode d'apprentissage de la lecture mixte en y intégrant les composantes des deux méthodes traditionnelles. Elles utilisent simultanément la méthode globale, dans laquelle les enfants sont conduits à apprendre les mots dans la forme lexicale précédée par leur article, ainsi qu'une méthode alphabétique-phonologique, qui incite les enfants à faire des liens entre les graphèmes et les phonèmes et à travailler sur la conscience métaphonologique, par exemple, à reconnaitre le même son dans différents mots. Les enfants sont encouragés à connecter graphème-phonème et mots par des tableaux accrochés sur leur table et sur les murs de la classe. De plus, ils se servent d'un outil appelé «cahier phonologique » dans lequel ils regroupent les mots écrits qui ont le même son initial. Par exemple, toutes les fois qu'ils retrouvent un mot qui commence avec le son [J], ils doivent l'écrire dans la page dédiée au son [J]. À la fin, dans cette page, nous retrouvons une liste de formes orthographiques (ex. château, chapeau, choix, etc.).

12 Les 43 enfants ont été testés vers la fin de l'année scolaire (mai 2014) au moyen d'une tâche d'écriture et une tâche de lecture à voix haute.

\subsection{Répertoire}

13 Pour sélectionner le répertoire de la tâche de lecture et de la tâche d'écriture, afin de tenir compte des vraies connaissances lexicales des enfants et pour avoir accès aux contextes syntaxiques dans lesquels les enfants ont appris les mots (savoir s'ils les ont rencontrés de façon isolée ou en contexte de liaison, d'élision ou d'enchainement), nous avons créé notre propre base de données du lexique écrit des enfants du CP. Nous avons utilisé pour la création deux cahiers fournis par les professeures des écoles, utilisés depuis septembre 2013, dans les heures de langue française. Le premier cahier est utilisé par les enfants à l'école avec les professeures. Il contient des exercices qui aident à développer le rapport entre graphème et phonème (comme, par exemple, rechercher le même son dans différents mots écrits). L'autre cahier, utilisé à la maison avec les parents, rassemble des devoirs dont le but est la répétition de la leçon et le renforcement des connaissances apprises en classe. Dans ce cahier, nous observons des petites comptines qui poussent les élèves à travailler de manière autonome sur un son spécifique.

14 Dans les cahiers, nous retrouvons ces mots dans 3 contextes : déterminant+nom (ex. les amis ou une maison), adjectif+nom (ex. fausse statue) et en isolation (ex. igloo). Pour construire une liste complète du lexique des enfants à utiliser comme répertoire, nous avons traité les mots présentés aux enfants avec des déterminants différents comme des entrées différentes. Par exemple, nous avons considéré le même item nez comme deux 
formes différentes le nez et un nez, car elles sont précédées d'un déterminant différent. Ces différenciations sont très importantes pour les noms qui commencent par une voyelle, car il est fondamental de voir si les mots ont été appris en contexte de liaison, d'élision ou d'enchainement ou s'ils ont plutôt été stockés en isolation. Comme nous l'avons déjà dit, en présence de phénomènes de resyllabation, la consonne finale de Mot1 est resyllabifiée au début de Mot2. Les enfants ayant écrit ou lu le Mot2 seulement en forme isolée peuvent avoir des difficultés à l'enchainer avec un déterminant spécifique en liaison, élision ou enchainement. De plus, réussir à combiner avec un déterminant différent le nom appris avec un certain déterminant peut créer des problèmes. Par exemple, s'ils ont seulement vu l'orthographe de <l'éléphant>, ils peuvent avoir des difficultés à écrire <un éléphant>. Ils risquent plutôt de produire des formes comme <un l'éléphant>. Comprendre et classifier les contextes dans lesquels les enfants ont appris la forme écrite de Mot2 est fondamental pour notre recherche.

Sur la base de cette méthode, en considérant comme deux formes séparées deux mots utilisés avec des déterminants différents, notre base de données compte 855 formes. Les 855 formes ont été classées par ordre alphabétique.

Après avoir classifié les formes orthographiques d'un ou deux mots, nous avons regardé tous les noms communs commençant par des voyelles. Nous avons créé une liste d'items à voyelle initiale en associant une fréquence. La fréquence que nous avons indiquée est également la fréquence absolue, le nombre de fois où l'item a été repéré dans les cahiers, sans tenir compte de la séquence Mot1+Mot2 où il est contenu. Par exemple, l'item ananas a une fréquence de 2. Cela veut dire que nous pouvons trouver ce mot deux fois dans les deux cahiers des enfants.

La fréquence moyenne dans la liste des items est de 2. Pour choisir les mots à utiliser dans les tâches liées à la langue écrite, nous avons pris les items dont la fréquence moyenne est au-dessus de la moyenne (fréquence absolue supérieure à 2). Nous avons choisi de prendre des mots avec une fréquence supérieure à la moyenne pour rendre les tâches liées à la langue écrite plus accessibles au $\mathrm{CP}$, car écrire et lire ces mots, qui n'ont pas une orthographe transparente, peut déjà être une source de problèmes. Proposer d'orthographier des mots de fréquence moyenne ou au-dessus de la moyenne comme Mot2 de nos séquences tests peut donc combler le fait que nous n'avons pris en considération ni la transparence des séquences à écrire ou à lire ni d'autres critères morpho-phonologiques (comme le nombre de syllabes de Mot2 ou l'orientation en nombre du nom).

Nous avons combiné les noms communs (Mots2) choisis dans 20 séquences tests déterminant+nom (Mot1+Mot2), divisés en 5 catégories différentes :

- 4 séquences de liaison au singulier (liaison en [n], ex. un oiseau);

- 4 séquences de liaison au pluriel (liaison en [z], ex. les ours);

- 4 séquences d'élision (élision, ex. l'école);

- 4 séquences d'enchainement (enchainement en [n], ex. une ambulance);

- 4 séquences à $h$ initial (2 séquences déterminant-nom avec $h$ aspiré, ex. un hippopotame et 2 séquences avec $h$ muet, ex. un hérisson).

Pour chaque phénomène, nous avons 4 séquences dont 2 présentées exactement dans les contextes déterminant+nom que nous avons repérés dans les cahiers. Les deux autres séquences sont composées par un déterminant qui permet la réalisation du phénomène de resyllabation et par un Mot2 que les enfants ont appris en isolation. De cette façon, 
10 séquences tests sont présentées dans ce que nous pouvons appeler «contextes connus ", des contextes que les enfants ont écrit et lu dans les cahiers comme des suites de Mot1+Mot2. Les 10 autres séquences sont présentées dans des contextes inconnus où Mot1+Mot2 n'ont jamais été appris ensemble. Ce choix nous permet d'évaluer la variable «familiarité »: les mots présentés dans un contexte connu sont des séquences plus familières aux enfants que les mots présentés dans un contexte inconnu.

Les Mots2 choisis pour la création de ces séquences présentent des valeurs variées de l'Indice de Fréquence Standard (SFI) dans la base de données du lexique écrit des enfants au CP, MANULEX (Leté, Sprenger-Charolles \& Colé, 2004). L'indice SFI est calculé à partir de l'estimation de la fréquence sur un million de mots $(U)$, par transformation logarithmique au moyen de la formule $:$ SFI $=10 *(\log 10(U)+4)$. Contrairement aux valeurs de la fréquence dans notre base de données présentées précédemment, SFI correspond à un indice standardisé qui a été statistiquement équilibré par l'opération du logarithme. Pour cette raison, nous avons décidé d'évaluer le rôle de la fréquence en utilisant l'indice SFI.

21 Nous présentons les séquences tests, la familiarité (contextes connus et inconnus) et les valeurs de SFI en annexe 1.

Il faut remarquer que nous avons classifié les contextes par familiarité (contextes connus et inconnus) selon la base de données de l'écrit créée au moyen des items utilisés dans les activités d'écriture et de lecture rédigées par les professeures des écoles. Nous ne pouvons pas tenir compte des activités externes à la classe où les enfants peuvent accéder aux formes écrites (ex. lecture partagée avec leurs parents au moyen des livres illustrés).

La tâche de lecture a eu lieu une semaine après la tâche d'écriture. Le délai d'une semaine entre les deux tâches nous garantit d'annuler les éventuels effets d'amorçage (Ferrand, 2009). De plus, pendant la tâche d'écriture, nous ne donnons pas aux enfants de retours sur la forme écrite correcte qu'ils doivent orthographier. Par conséquent, nous n'avons pas mis à disposition des enfants des informations sur les productions écrites correctes qui peuvent influencer la tâche de lecture et leur laisser des traces en mémoire.

\subsection{Procédure}

\subsubsection{La tâche d'écriture}

Pour la tâche d'écriture, nous avons divisé les 43 enfants en 4 groupes : deux groupes de 10 , un groupe de 11 et un groupe de 12 , en respectant la classe d'appartenance. Chaque groupe a été testé dans une salle de classe isolée et silencieuse. Pour chaque élève, nous avons créé un petit livret relié au sein duquel chacune des pages contient une image dans un carré. En dessous de chaque image se trouve une ligne pointillée que les enfants doivent utiliser pour écrire.

L'expérimentateur nomme l'image qui apparait dans la page du livret en prononçant la séquence test, ex. les ours [le.zurs]. Les enfants doivent donc écrire sur la ligne pointillée, au-dessous de l'image, les deux mots produits (les ours) qui correspondent aux séquences tests de notre répertoire. Quand tout le monde a terminé d'écrire la première séquence, les enfants doivent tourner la page, et écrire sur la ligne pointillée la deuxième séquence test prononcée par l'expérimentateur. La tâche se termine quand le livret est complété et que les 20 séquences tests sont écrites. En annexe 2, nous présentons un modèle du livret donné. La tâche durait environ 45 minutes à l'issue de laquelle l'examinateur ramassait 
les livrets. L'ordre de présentation du répertoire variait selon le groupe pour éviter des effets de facilitation ou de stratégies.

\subsubsection{La tâche de lecture}

26 Les enfants sont testés un par un au moyen d'une tâche de lecture à voix haute. Nous avons imprimé les 20 séquences tests (en minuscules, police Times New Roman, taille 25) utilisées pour la tâche d'écriture sur 20 cartes $(5 \times 10 \mathrm{~cm})$. Nous montrons à chaque enfant une carte avec la séquence écrite et nous lui demandons de la lire. Dès qu'il a terminé la lecture de la carte, nous proposons la carte suivante et nous invitons à nouveau à la lecture. La tâche se termine quand les 20 séquences tests écrites sur les 20 cartes sont lues. La lecture des séquences a été enregistrée au format .wav par un enregistreur Zoom $\mathrm{H} 4$ à une fréquence de $44000 \mathrm{~Hz}$. L'ordre de présentation du répertoire variait selon l'enfant pour éviter des effets de facilitation ou de stratégies.

\section{Résultats}

\subsection{La tâche d'écriture}

Tous les enfants écrivent les mots dictés en écriture liée. Nous avons considéré seulement 42 enfants dans les données, car nous avons écarté les résultats d'un enfant $(2,3 \%)$ incapable d'écrire. Nous avons également décidé d'analyser seulement 16 contextes (liaison, élision et enchainement avec des Mots2 à voyelle initiale), en excluant les données concernant les contextes à $h$ muet ou $h$ aspiré initiales qui doivent être traités d'une façon différente et seront le sujet d'une future publication.

Notre recherche est centrée sur les phénomènes de resyllabation. Par conséquent, nous avons décidé d'exclure de notre analyse les erreurs orthographiques à l'intérieur des mots (ex. <ambulanse> pour <ambulance>; <otruche> pour <autruche>). Nous nous sommes concentrés sur le traitement de la frontière gauche de Mot2 qui nous a permis de classifier les productions écrites des enfants selon deux modalités possibles:

- Segmentations correctes : les séquences en liaison, élision et enchainement sont écrites en respectant les frontières lexicales des mots (ex. pour la liaison <les ours>, pour l'élision $<$ l'éléphant>, pour l'enchainement <une autruche>) ;

- Erreurs de segmentation: la consonne qui resyllabifie sur le Mot2 est traitée comme la consonne initiale de Mot2, en respectant les frontières syllabiques (ex. pour la liaison <les sours>, pour l'élision <le léléphant>, pour l'enchainement <une nautruche>).

$\mathrm{Au}$ moyen d'un $t$-test utilisant les sujets $\left(t_{1}\right)$ et les items $\left(t_{2}\right)$ comme variables indépendantes, nous observons qu'il y a une différence statistiquement significative entre le nombre des segmentations correctes et le nombre des erreurs de segmentation $\left(t_{1}\right.$ $\left.(41)=3,472, p<0,001 ; t_{2}(15)=2,79, p<0,05\right)$. La plupart des enfants réussissent à écrire correctement les mots, en respectant les frontières lexicales perturbées par les phénomènes de resyllabation à l'oral (graphique 1). Pour plus de détails, nous présentons les scores moyens de segmentations correctes et de segmentations erronées en annexe 3. 
Graphique 1. - Scores moyens de productions écrites (segmentations correctes et erreurs de segmentation). Le score maximal est 16.

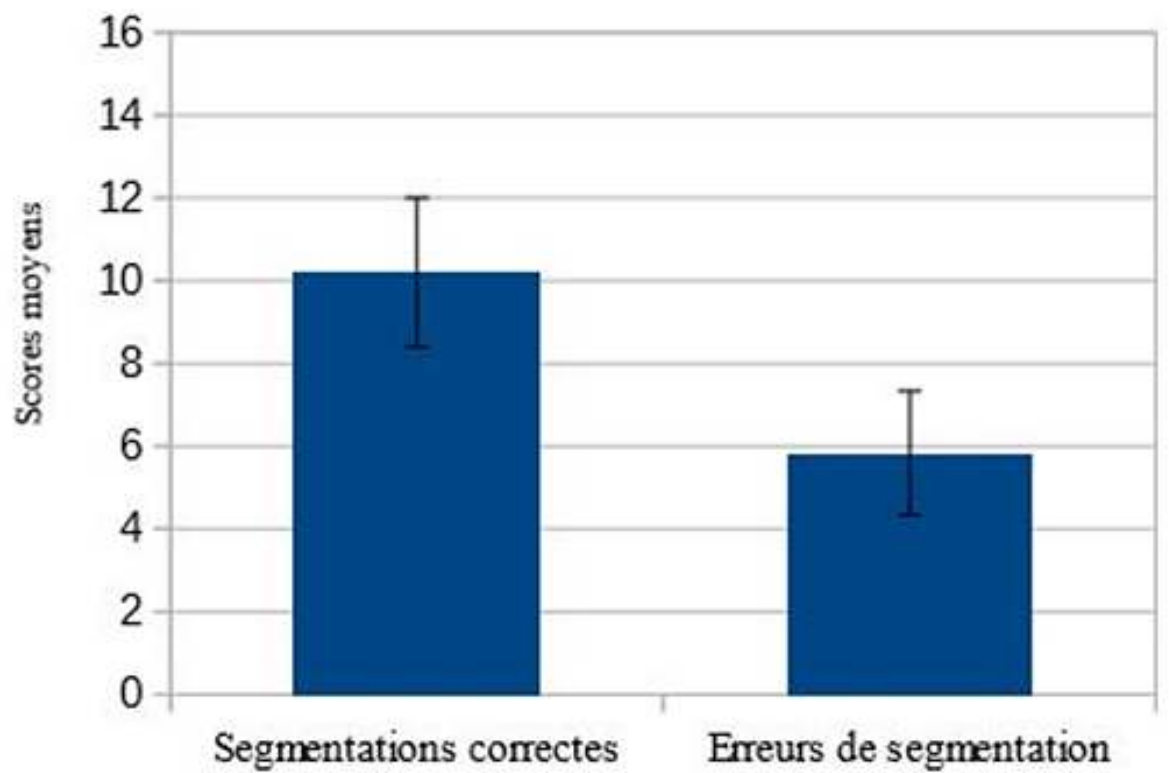

Cependant nous remarquons encore beaucoup d'erreurs de segmentation à l'écrit $(M=5,86$, écart-type $=1,5)$. Nous avons donc décidé de vérifier s'il existe une différence statistiquement significative pour les scores d'erreurs de segmentation (ex. <une notruche>, <lécole> et <les zours>) dans les deux types de contextes (séquences de Mot1 +Mot2 connus et séquences de Mot1+Mot2 inconnus) prévus par le répertoire. Le résultat $\mathrm{du}$ t-test utilisant les sujets $\left(\mathrm{t}_{1}\right)$ et les items $\left(t_{2}\right)$ comme variables indépendantes montre une différence statistiquement significative $\left(t_{1}(41)=2,672, p<0,001 ; t_{2}(7)=1,98\right.$, $\mathrm{p}<0,05)$ : nous observons plus d'erreurs de segmentation à l'écrit pour les contextes inconnus (graphique 2). Cela veut dire que les enfants ont plus de difficultés à orthographier une séquence de Mot1 suivi par un Mot2 s'ils n'ont jamais vu ces deux mots l'un après l'autre. 
Graphique 2. - Scores d'erreurs à l'écrit (contextes connus et contextes inconnus). Le score maximal d'erreurs de segmentation est 8.

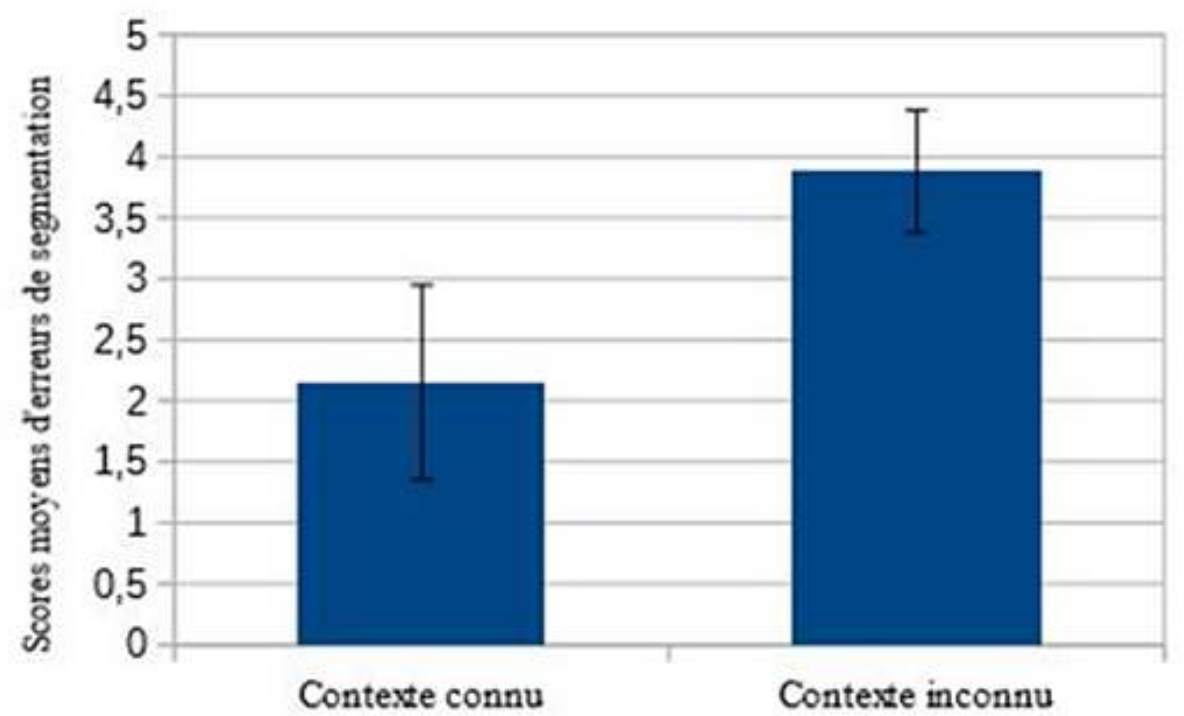

Après avoir analysé la familiarité avec les contextes, nous évaluons l'influence de la fréquence de Mot2 sur les productions écrites des enfants. Nous établissons un rapport entre les valeurs de la fréquence des Mots2 au CP dans la base de données du lexique écrit des enfants MANULEX (indice SFI) et les scores d'erreurs de segmentation à l'écrit (ex. <les sours>), au moyen du calcul de l'indice de corrélation $r$ de Pearson. Le résultat montre qu'il n'y a pas de corrélation entre ces deux variables $(r(15)=0,29, p=0,25)$. La frontière gauche d'un Mot2 qui apparait plus fréquemment à l'écrit n'est pas mieux identifiée et orthographiée que la frontière gauche d'un Mot2 moins fréquent. La seule fréquence de Mot2 ne permet pas de mieux orthographier ce mot et d'identifier sa frontière gauche à l'écrit.

\subsection{La tâche de lecture à voix haute}

Pour la tâche de lecture, nous avons écarté 2 enfants (4,6 \%) qui n'ont pas été capables de lire les mots. Nous considérons les scores de lectures correctes pour lesquelles les phénomènes de resyllabation sont bien réalisés, car la consonne impliquée est bien resyllabifiée sur Mot2 ([le.zubs], [le.kol], [y.nõ.by.lõs]) et les scores de lectures erronées. Nous avons analysé phonétiquement ces lectures erronées au moyen du logiciel Praat (Boersma \& Weenink, 2013) et nous avons remarqué trois types de productions :

- une véritable pause est produite entre Mot1 et Mot2 (ex. le(s)-pause-affiches [le/a.fij]; un-

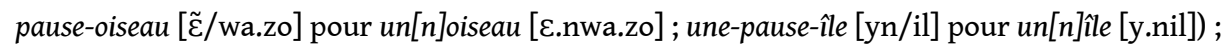

- une pause est produite après Mot1 et un Mot2 à attaque remplie est réalisé (ex. les-pause-[z ]affiches [le/za.fi]] pour les[z]affiches [le.za.fi]]; le-pause-[l]éééphant [lə/le.le.fã] pour l'éléphant [le.le.fã] ; une-pause-[n]île [yn/nil] pour un[n]île [y.nil]);

- entre Mot1 et Mot2, une occlusive glottale est insérée (ex. le(s)[?]affiches [le.Pa.fij] pour les [z]affiches [le.za.fi]]; une[?] île [yn.Pil] pour une[n]île [y.nil]).

Nous comparons les lectures correctes et erronées au moyen d'un t-test utilisant les sujets $\left(t_{1}\right)$ et les items $\left(t_{2}\right)$ comme variables indépendantes. Le résultat montre une 
différence statistiquement significative entre les deux scores $\left(t_{1}(40)=8,108, p<0,001 ; t_{2}\right.$ $(15)=6,365, p<0,001)$. Les enfants produisent plus de lectures correctes que de lectures erronées (graphique 3). Pour plus de détails, nous présentons les scores moyens de lectures correctes et de lectures erronées en annexe 4.

Graphique 3. - Scores moyens pour la lecture à voix haute (lectures correctes et lectures erronées). Le score maximal est 20.

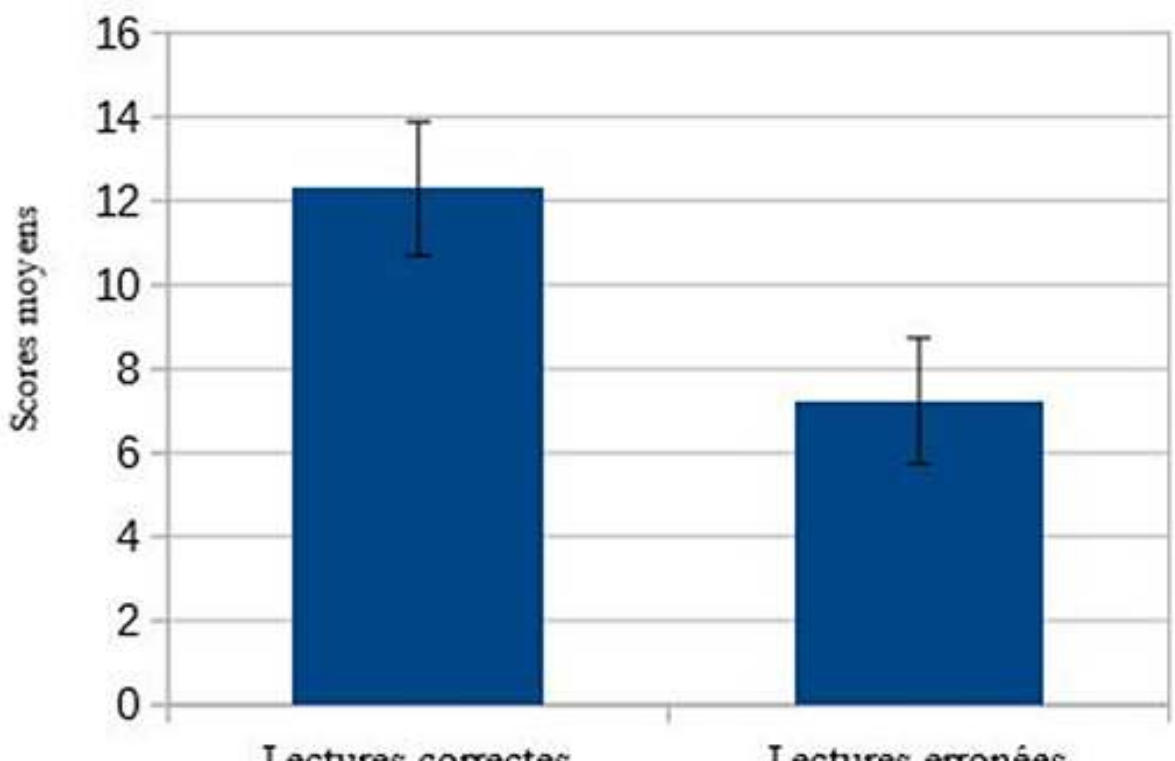

Nous avons décidé d'analyser les scores de lectures erronées dans les deux contextes (connus et inconnus) au moyen d'un t-test en utilisant les sujets $\left(t_{1}\right)$ et les items $\left(t_{2}\right)$ comme variables indépendantes. Le t-test indique qu'il n'y a pas de différence statistiquement significative pour les scores de lectures erronées entre les contextes connus et les contextes inconnus $\left(t_{1}(40)=0,65, p=0,51 ; t_{2}(7)=0,67, p=0,43\right)$. La familiarité à l'écrit avec un certain type de contexte n'intervient pas dans le processus de la lecture des séquences en liaison, en élision ou en enchainement.

Nous calculons également la corrélation entre la fréquence à l'écrit des Mots2 dans le lexique des enfants (SFI dans MANULEX) et les scores de lectures erronées. Le calcul du coefficient $r$ de Pearson permet d'établir une corrélation négative entre les scores de lectures erronées et les valeurs de la fréquence $(r(15)=-0,61, p<0,05)$. Nous observons une diminution d'erreurs en rapport avec l'augmentation de la fréquence des Mots2 à lire et vice-versa. Cela veut dire qu'un Mot2 plus fréquent dans le lexique des enfants est mieux lu qu'un Mot2 moins fréquent et qu'il est aussi plus facile de produire les phénomènes de resyllabation quand ce mot est fréquent.

\section{Discussion / conclusions}

Les résultats de la tâche d'écriture et de la tâche de lecture nous montrent que la plupart des enfants orthographient et lisent correctement les séquences de Mot1+Mot2 à la fin du CP. Les données de la tâche d'écriture infirment nos hypothèses, car dans la plupart 
des cas, ils réussissent à identifier les frontières de Mot2 à l'écrit sans être influencés par les phénomènes de resyllabation. une fréquence variée et ils sont insérés dans différents contextes : la moitié des contextes testés est connue par les élèves et l'autre moitié n'est pas connue par les élèves. Les contextes connus sont traités en classe par les professeures des écoles qui proposent des travaux de lecture et d'écriture sur ces séquences. Les contextes inconnus ne sont pas sujets à un apprentissage explicite à l'école. Le choix de ces deux contextes fait émerger la variable «familiarité »: dans les contextes connus, le niveau de familiarité des enfants avec les séquences est très élevé, car les enfants ont déjà vu la suite Mot1+Mot2 à l'écrit. Cela n'est pas le cas pour les contextes inconnus parce que les élèves n'ont jamais vu à l'école ces deux mots l'un après l'autre. Nous remarquons pour les deux tâches des résultats différents sur les deux contextes que nous avons proposés et également un rapport différent avec la fréquence de Mot2. Dans la tâche d'écriture, les contextes les mieux écrits sont les contextes connus, où la frontière vocalique gauche de Mot2 est identifiée plus facilement. De plus, la variable « fréquence » n'influence pas l'orthographe de Mot2 : la frontière gauche de Mot2 n'est pas mieux identifiée ou orthographiée si ce mot est un item fréquent dans le lexique écrit des enfants. En revanche, dans la tâche de lecture, nous n'enregistrons pas de différences dans le traitement des contextes connus et des contextes inconnus, mais nous observons un effet modéré de la fréquence. Un Mot2 plus fréquent dans le lexique écrit des enfants est mieux lu et enchainé au mot qui précède qu'un Mot2 moins fréquent.

À la lumière de ces résultats, nous pouvons dire que si les enfants ont déjà vu une certaine séquence déterminant-nom, caractérisée par un phénomène de resyllabation à l'écrit et qu'ils ont, par conséquent, une représentation disponible de cette séquence, ils ont moins 
de problèmes à déterminer sur papier les frontières des deux mots qui la composent. Au contraire, la connaissance des suites Mot1+Mot2 n'apporte pas d'avantages dans la lecture de ces séquences et dans la réalisation des phénomènes de resyllabation insérés. La fréquence de Mot 2 joue un rôle dans la lecture en favorisant les réalisations correctes des phénomènes de resyllabation, car un Mot2 plus fréquent est plus facilement enchainé au Mot1 qui précède. La familiarité avec les séquences Mot1+Mot2 est donc déterminante pour la segmentation à l'écrit à ce stade, ainsi que la fréquence de Mot2 pour la lecture des séquences resyllabifiées. Ces résultats confirment l'idée que lecture et écriture sont deux activités différentes qui font référence à des stratégies différentes (Cossu \& Marshall, 1985). Par conséquent, l'apprentissage doit être structuré selon l'activité que l'enseignant veut renforcer. Pour bien apprendre à orthographier les frontières gauches des Mots2 en présence des phénomènes de resyllabation, il est préférable d'utiliser une méthode d'apprentissage qui propose la visualisation et la production écrite des suites Mot1+Mot2, plutôt qu'une liste de Mots1 en isolation. Pour bien lire les phénomènes de resyllabation, il faut plutôt construire du matériel didactique qui alterne la présence de Mots2 isolés et de suites de Mot1+Mot2.

Dans la sélection du répertoire, nous avons décidé d'utiliser comme critère la familiarité des séquences dans les cahiers des enfants. Pour cette raison, nous n'avons pas pris en compte pour la création du répertoire de la longueur de Mot2 (le nombre de syllabes), le nombre de graphèmes/lettres et le type de graphème ou de phonème en initiale. Ces critères peuvent avoir des effets sur les résultats des tâches proposées. Par exemple, un Mot2 plus long ou qui présente un digramme à l'initiale peut causer des difficultés en lecture et en écriture au $\mathrm{CP}$. Une autre étude avec un répertoire plus homogène du point de vu phonologique et orthographique peut donc être envisagé. De plus, les études de Dugua, Spinelli, Chevrot et Fayol (2009) ont montré que, chez les enfants de 5-6 ans, l'orientation en nombre des noms influence la réalisation orale de la liaison. Par conséquent, nous pouvons penser que ce critère d'orientation a une incidence sur le traitement de la liaison en lecture chez de jeunes apprenants. Étudier la lecture et l'écriture des phénomènes de resyllabation en rapport avec l'orientation en nombre des noms serait le but de recherches futures.

\section{BIBLIOGRAPHIE}

BABINEAU, Mireille \& SHI, Rushen. (2011). Processing of French Liaisons in Toddlers. Dans BUCLD 35: Proceedings of the 35th Annual Boston University Conference on Language Development 1 (p. 25-37). Boston, MA : Cascadilla Press.

BÉCHADE, Hervé-D. (1992). Phonétique et morphologie du français moderne et contemporain. Paris, France : Presses universitaires de France.

Blanche-Benveniste, Claire \& Jeanjean, Colette. (1987). Le français parlé : transcription et édition. Paris, France : Didier Érudition.

Boersma, Paul \& WeEninK, David. (2013). Praat: doing phonetics by computer. [LOGICIEL] 
CHETAIL, Fabienne. (2012). La syllabe en lecture. Rennes, France : Presses universitaires de Rennes. Chevrot, Jean-Pierre, Dugua, Céline, Harnois-DelPiano, Mylène, Siccardi, Anne \& SPInelli, Elsa. (2013). Liaison Acquisition: Debates, Critical Issues, Future Research. Language Sciences, 39, 83-94. ColÉ, Pascale, MAGnAN, Annie \& GRAINGER, Jonathan. (1999). Syllable-sized Units in Visual Words Recognition: Evidence from Skilled and Beginning Readers. Applied Psycholinguitics, 20, 507-532.

Cossu, Giuseppe \& MARSHALL, John C. (1985). Dissociation between Reading and Written Spelling in Two Italian Children. Dyslexia without Dysgraphia? Neuropsychologia, 23, 697-700.

Delattre, Pierre. (1951). Principes de phonétique française à l'usage des étudiants anglo-américains. Middlebury, Vermont : Middlebury College.

Delattre, Pierre. (1965). Comparing the Phonetic Features of English, French, German and Spanish. Heidelberg, Allemagne : Jumius Gross Verlag.

Di CRISTO, Albert. (1998). Intonation in French. Dans D. Hirst \& A. Di Cristo (dir.), Intonation Systems: A Survey of Twenty Languages (p. 195-218). Cambridge, Royaume-Uni : Cambridge University Press.

DuGUA, Céline. (2006). Liaison, segmentation lexicale et schémas syntaxiques entre 2 et 6 ans. Un modèle développemental basé sur l'usage (Thèse de doctorat). Université Stendhal-Grenoble 3, Grenoble.

Dugua Céline \& ChABANAL, Damien (2006). Acquisition de la liaison chez l'enfant francophone, formes lexicales des mots2. Poster lors du colloque International « JEP (Journées d'études sur la parole), Dinard, France. [CD-Rom]

Dugua, Céline, Spinelli, Elsa, Chevrot, Jean-Pierre \& FAyol, Michel (2009). Usage-based Account of the Acquisition of Liaison: Evidence from Sensitivity to the Singular/Plural Orientation of Nouns. Journal of Experimental Child Psychology, 102, 342-350.

Dugua, Céline \& BACLESSE, Marie. (2014). Incidence d'effets de fréquence sur l'usage de la liaison en lecture à haute voix et dans des jugements normatifs chez des enfants de CE2-CM1. Dans C. Soum-Favaro, A.-L. Coquillon \& J.-P. Chevrot (dir.), La liaison : approches contemporaines (p. 117-139). Berne, Suisse : Peter Lang.

ENCREVÉ, Pierre. (1983). La liaison sans enchaînement. Actes de la recherche en sciences sociales, 46, 39-66.

ENCREVÉ, Pierre. (1988). La liaison avec et sans enchaînement : phonologie tridimensionnelle et usage du français. Paris, France : Seuil.

FERRAND, Ludovic (2009). Psychologie cognitive de la lecture : Reconnaissance des mots écrits chez l'adulte . Bruxelles, Belgique : De Boeck.

GoswAmi, Usha \& BRYANT, Peter. (1990). Phonological Skills and Learning to Read. Londres, Royaume-Uni : Erlbaum.

Goswami, Usha. (1993). Toward an Interactive Analogy Model of Reading Development: Decoding Vowel Graphemes in Beginning Reading. Journal of Experimental Child Psychology, 56, 443-475.

GoswamI, Usha. (1999). Causal Connections in Beginning Reading: The Importance of Rhyme. Journal of Research in Reading, 22, 217-240.

KANDEL, Sonia \& VALDOIS, Sylviane. (2006). French and Spanish Speaking Children Use Different Units to Read and Write. Language and Cognitive Processes, 21(5), 531-561. 
KAYE, Jonathan \& LoWEnSTAMm, Jean. (1984). De la syllabicité. Dans F. Dell, D. Hirst \& J.-R. Vergnaud (dir.), Formes sonores du langage : structure de représentation en phonologie (p. 123-159). Paris, France : Hermann.

LÉTÉ, Bernard, SPRENGER-CHARolles, Lilliane \& ColÉ, Pascale. (2004). MANULEX: A Grade-level Lexical Database from French Elementary School Readers. Behavior Research Methods, 36(1), 156-166.

NARDY, Aurélie \& DuguA, Céline. (2011). Le rôle de l'usage sur le développement des constructions nominales chez les enfants pré-lecteurs. Travaux de linguistique, 1, 129-148.

NazZI, Thierry, IAKImova, Galina, Bertoncini, Josiane, Fredonie, Séverine \& AlCANTARA, Carmela. (2006). Early Segmentation of Fluent Speech by Infants Acquiring French: Emerging Evidence for Crosslinguistic Differences. Journal of Memory and Language, 54(3), 283-299.

NAzzI, Thierry. (2008). Segmentation précoce de la parole continue en mots : évaluation interlinguistique de l'hypothèse d'initialisation rythmique. L'Année psychologique, 108, 309-342.

Pulgram, Ernst. (1970). Syllable, Word, Nexus, Cursus. The Hague, Pays-Bas : Mouton.

Soum, Christiane (1997). L'apprentissage de l'écriture ; contraintes orthographiques; contraintes orales (Thèse de doctorat). Université Toulouse 2, Toulouse.

Soum-Favaro, Christiane, Gunnarsson, Cécilia \& LARgy, Pierre. (2014). La liaison à l'interface entre l'oral et l'écrit. Dans C. Soum-Favaro, A.-L. Coquillon \& J.-P. Chevrot (dir.), La liaison : approches contemporaines (p. 141-167). Berne, Suisse : Peter Lang.

WAUQUier-Gravelines, Sophie \& BRAUd, Virginie. (2005). Proto-déterminant et acquisition de la liaison obligatoire en français. Langages, 158, 53-65.

WAUQUIER, Sophie. (2009). Acquisition de la liaison en L1 et L2 : stratégies phonologiques ou lexicales? Aile...Lia, 2, 93-130.

WAUQUIER, Sophie \& SHOEMAKER, Ellenor. (2013). Convergence and Divergence in the Acquisition of French Liaison by Native and Non-native Speakers: A Review of Existing Data and Avenues for Future Research. Language, Interaction and Acquisition, 4(2), 161-189.

ZIEGLER, Johannes C. \& Goswami, Usha (2005). Reading Acquisition, Developmental Dyslexia and Skilled Reading across Languages: A Psycholinguistic Grain Size Theory. Psychological Bulletin, 131 (1), 3-29.

\section{ANNEXES}

Annexe 1. - Matériel utilisé pour les tâches d'écriture et de lecture à voix haute. 


\begin{tabular}{|c|c|c|c|}
\hline Phénomènes & Familiarité & Séquences tests & $\begin{array}{l}\text { Valeurs de SFI } \\
\text { pour Mot2 } \\
\text { (MANULEX) }\end{array}$ \\
\hline \multirow{4}{*}{ Liaison en $[z]$} & \multirow{2}{*}{ Connu } & Les ours [le.zurs] & 68,21 \\
\hline & & Des ailes [de.zel] & 61,42 \\
\hline & \multirow{2}{*}{ Inconnu } & Les oranges [le.zo.Rãz] & 59,09 \\
\hline & & Les affiches [le.za.fif] & 58,83 \\
\hline \multirow{4}{*}{ Liaison en [n] } & \multirow{2}{*}{ Connu } & Un oiseau [r.nwa.zo] & 69,25 \\
\hline & & 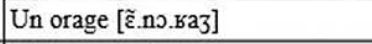 & 59,09 \\
\hline & \multirow{2}{*}{ Inconnu } & Un igloo [z̃.ni.glu] & 49,28 \\
\hline & & Un ananas [z̃.na.na.nas] & 58,02 \\
\hline \multirow{4}{*}{ Élision } & \multirow{2}{*}{ Connu } & L'oie [lwa] & 61,07 \\
\hline & & L'école [le.kol] & 70,40 \\
\hline & \multirow{2}{*}{ Inconnu } & L'âne [lan] & 62,74 \\
\hline & & L'éléphant [le.le.fã] & 65,99 \\
\hline \multirow{4}{*}{ Enchaînement } & \multirow{2}{*}{ Connu } & Une épée [y.ne.pe] & 58,40 \\
\hline & & Une autruche [y.not.byf] & 56,75 \\
\hline & \multirow{2}{*}{ Inconnu } & Une ambulance [y.nã.by.lãs] & 56,59 \\
\hline & & Une île [y.nil] & 59,00 \\
\hline \multirow{2}{*}{$h$ aspiré } & Inconnu & 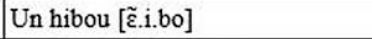 & 59,30 \\
\hline & Connu & 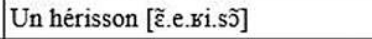 & 61,03 \\
\hline \multirow{2}{*}{$h$ muet } & Connu & Un hippopotame [r.ni.po.po.tam] & 56,73 \\
\hline & Inconnu & Une hirondelle [y.ni..õ.dal] & 57,82 \\
\hline
\end{tabular}

Annexe 2. - Exemple du livret utilisé pour la tâche d'écriture (9 pages sur 20).
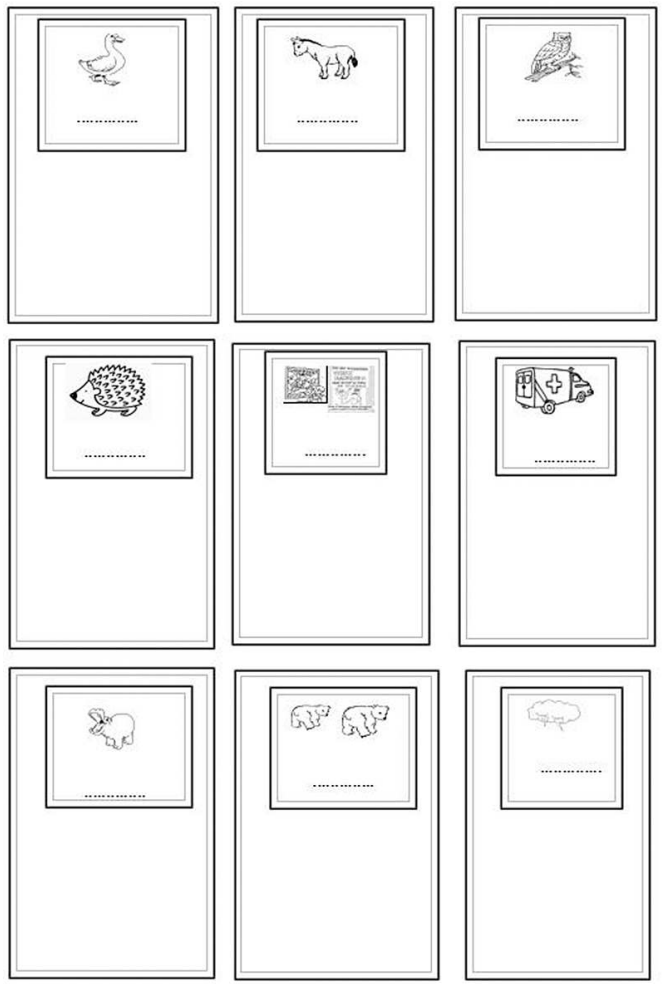

Annexe 3. - Résultats de la tâche d'écriture pour les 4 phénomènes traités dans l'analyse de données (liaison en [n], liaison en [z], élision et enchainement). Le score maximal est 4. 


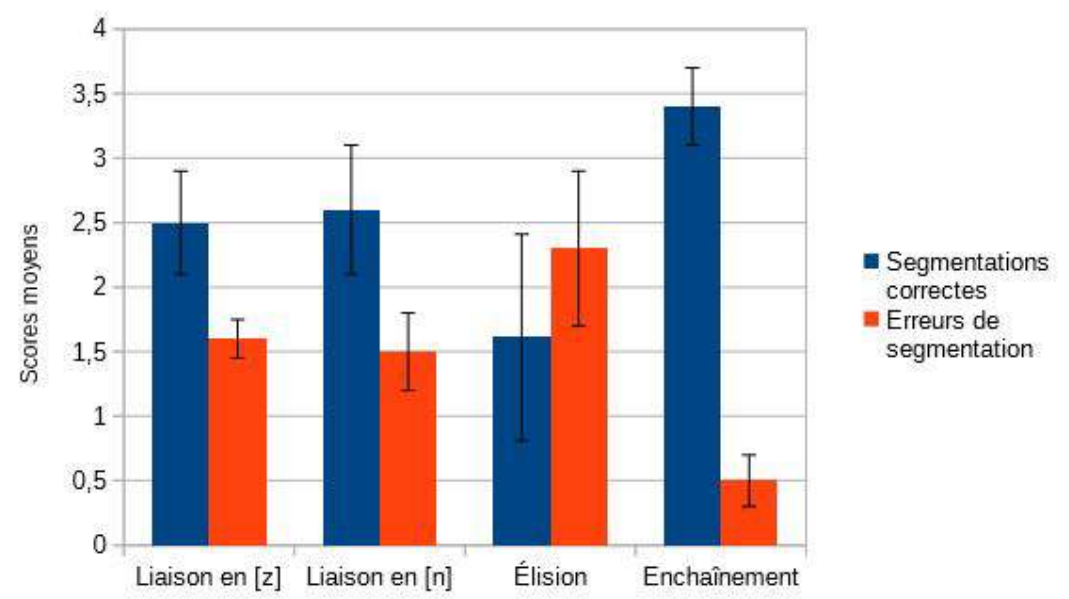

Le résultat des analyses montre une différence statistiquement significative entre les scores de segmentations correctes et de segmentations erronées pour la liaison en [z], en [n] et pour l'enchainement (liaison en $[z]: t_{1}(40)=1,35, p<0,05, t_{2}(3)=1,29, p<0,05$; liaison en $[n]: t_{1}(40)=1,052, p<0,001, t_{2}(3)=1,298, p<0,001$; enchainement : $t_{1}$ $\left.(40)=2,887, p<0,001, t_{2}(3)=2,967, p<0,001\right)$. Pour ces trois phénomènes de resyllabation, les enfants segmentent correctement les séquences à l'écrit (plus de segmentations correctes que d'erreurs en segmentation). Pour l'élision, nous n'observons pas de différence significative entre les scores de segmentations correctes et de segmentations erronées $\left(t_{1}(40)=0,71, p=0,79 ; t_{2}(3)=0,58, p=0,83\right)$. Le phénomène qui pose le plus de difficultés à l'écrit est donc l'élision. En analysant les erreurs pour ce phénomène, dans la plupart des cas $(80 \%)$ nous enregistrons des productions écrites présentant ce qu'on a appelé « hyposegmentation » : le déterminant et nom sont traités comme une seule unité et la consonne <l> devient l'initiale du Mot2 (ex. <loie> pour <l'oie>).

Annexe 4. - Résultats de la tâche de lecture à voix haute pour les quatre phénomènes traités dans l'analyse de données (liaison en [z], liaison en [n], élision et enchainement). Le score maximal est 4 . 


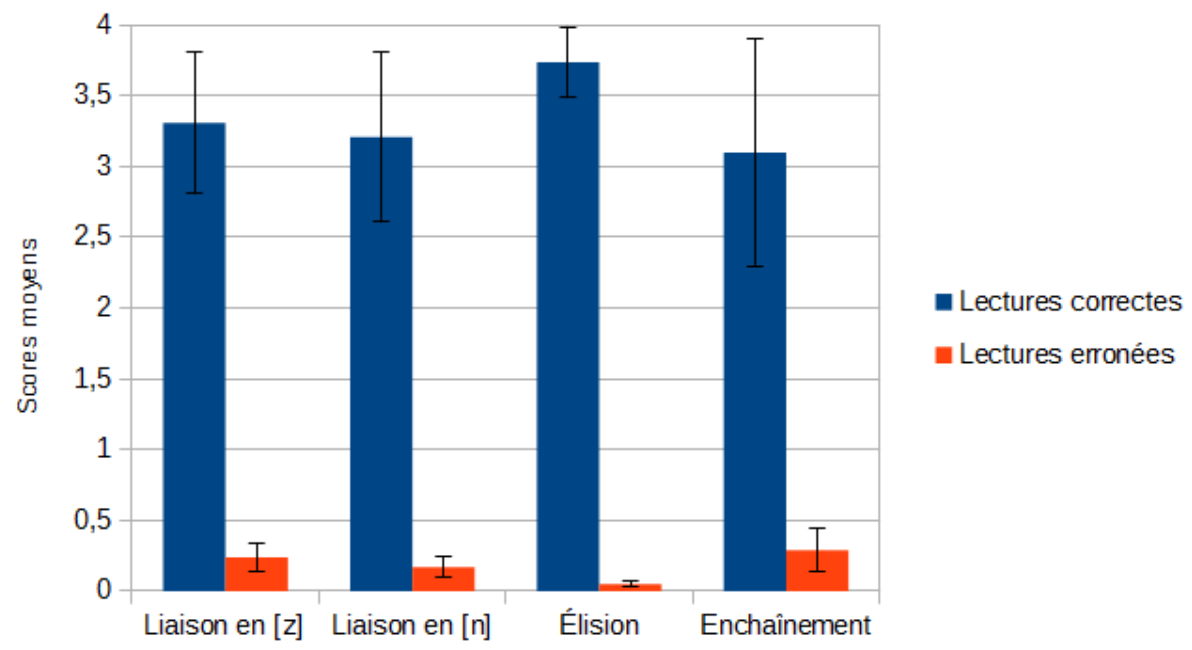

Le résultat montre une différence statistiquement significative entre les scores de lectures correctes et de lectures erronées pour tous les phénomènes (liaison en $[\mathrm{z}]: \mathrm{t}_{1}$ $(40)=-2,890, p<0,001, t_{2}(3)=2,201, p<0,001$; liaison en $[n]: t_{1}(40)=3,002, p<0,001, t_{2}$ (3) $=2,458, p<0,001$; élision : $t_{1}(40)=-2,931, p<0,001, t_{2}(3)=2,189, p<0,001$; enchainement : $\left.t_{1}(40)=3,689, p<0,001, t_{2}(3)=2,784, p<0,001\right)$. Les enfants produisent plus de lectures correctes que de lectures erronées pour les quatre phénomènes.

\section{RÉSUMÉS}

Pendant l'acquisition, l'enfant francophone reçoit comme input une langue orale dont les frontières lexicales sont masquées par trois phénomènes de resyllabation qui ont lieu dans des suites Mot1+Mot2 : la liaison (ex. Mot1 : les [le] + Mot2 : ami [ami] $\rightarrow$ les[z]ami [le.za.mi]), l'élision (ex. Mot $1:$ le [lə] + Mot $2:$ ours [uss] $\rightarrow$ l'ours [lubs]) et l'enchainement (ex. Mot1: une [yn] + Mot2: autruche [o.tKy $\left.\int\right] \rightarrow$ une[n]autruche [y.no.tKyf]). Ces phénomènes sont à l'origine de l'apparition de mauvaises segmentations dans les productions orales des enfants à partir de l'âge de 2 ans (ex. de(s)[n]ours [de.nurs] pour $\operatorname{des[z]ours~[de.zurs])~et~dans~les~premières~productions~}$ écrites à l'école primaire, surtout au CE1 (ex. <les sours>, <une nambulance>, <léléphant>). Dans cette étude, nous testons 43 enfants francophones au CP. Les résultats de deux tâches qui testent l'écriture et la lecture de séquences en liaison, en élision et en enchainement nous permettent de comprendre comment les enfants traitent les frontières de Mot2 à l'écrit à ce stade de l'acquisition de la littéracie, encore inexploré. De plus, le répertoire choisi permet d'analyser l'importance de la fréquence de Mot2 et le rôle de la familiarité avec les séquences de Mot1+Mot2 dans les activités de lecture et d'écriture. L'apprentissage de Mots2 insérés dans des suites Mot1 +Mot2 permet d'orthographier correctement les frontières lexicales. En revanche, proposer fréquemment des Mots2 isolés alternés aux suites Mot1+Mot2 permet de lire correctement les phénomènes de resyllabation.

Early word segmentation in French is complicated by three phenomena of re-syllabification: liaison (word1 les [le], 'the' + word2 amis [ami], 'friends' $\rightarrow$ les[z]amis [le.zami], 'the friends'), elision (word1 le [lə], 'the' + ours [urs], word2 'bear' $\rightarrow$ l'ours [luss], 'the bear') and enchainment 
(word1 une [yn], 'a' + word2 autruche [o.tьyf], 'ostrich' $\rightarrow$ une autruche [y.no.tbyf], 'an ostrich'). These processes of re-syllabification entail children's errors in oral productions (le $(s)$ [n]ours [le.nuss] for les[z]ours [le.zurs], 'the bears') and in writing forms in Second Grade (ex. <les sours>, <une nambulance>, <léléphant>). In this study, we test 43 French First Graders by a writing task and a reading task using liaison, elision and enchainment sequences. Firstly, we understand how French children starting Primary School deal with resyllabified sequences and literacy. Moreover, we evaluate if learning the writing form of word2 in the sequences word1+word2 is more convenient than learning word2 in isolation, thanks to the analysis of frequency and familiarity. The results of the writing task show that learning word+word2 writing forms helps to set lexical boundaries of word2 and write resyllabified sequences correctly. The results of the reading task tell us that it is also important to learn the form of word2 in isolation to perform correct reading.

\section{INDEX}

Mots-clés : liaison, segmentation, phénomènes de resyllabation, littéracie, fréquence, familiarité des séquences

Keywords : resyllabified sequences, segmentation, literacy, frequency, familiar sequences

\section{AUTEUR}

SAMANTHA RUVOLETTO

Université Paris 8, CNRS/UMR 7023 